\title{
A Pioneering Perusal of Mimo- OFDM Realy Guesstimate Disposition
}

\author{
${ }^{1}$ Manikanta, ${ }^{2}$ A. Vamsidhar \\ ${ }^{1}$ Student, M.tech, ${ }^{2}$ HOD Dept. of ECE \\ ${ }^{1,2}$ DADI Institute of Engineering \& Technology, Anakapalle
}

\begin{abstract}
A multiple-input multiple-output (MIMO) communication system combined with the orthogonal frequency partition multiplexing (OFDM) modulation technique can achieve reliable high data rate show over broadband wireless channels. Channel state information for both single-input single-output (SISO) and MIMO systems based on pilot aided arrangement is investigated in this paper. The estimate of channel at pilot frequencies with conventional Least Square (LS) and Minimum Mean Square (MMSE) estimation algorithms is carried out through Matlab simulation. For outdoor communicationscenarios, where wireless channels are sparse in nature, pathinterruptions of different transmit-receive antenna couples share a jointsparse decoration due to the spatial correlation of MIMO channels.By simultaneously exploiting those MIMOchannel characteristics, the proposed scheme performs better thanpresent state-of-the-art systems. Furthermore, by joint processing of signals associated with different antennas, the pilot overheadcan be reduced the performance of MIMO OFDM and SISO OFDM are assessed on the basis of Bit Error Rate (BER) and Mean Square Error (MSE) level. Further enhancement of presentation can be attained through maximum diversity Space Time Block Coding and Maximum Likelihood Detection at transmission and reception ends respectively. MMSE estimation has been shown to perform much better than LS but is more complex than LS for the MIMO system using pilot carriers.
\end{abstract}

Keywords: Antenna, BER, Detection, MIMO, OFDM, SISO, LS, MSE, MMSE

\section{Introduction}

Wireless technologies have evolved remarkably since Guglielmo Marconi first demonstrated radio's ability to provide continuous contact with ships sailing in the Englishchannel in 1897. New theories and applications of wireless technologies have beendeveloped by hundreds and thousands of scientists and engineers through the worldever since. Wireless communications can be regarded as the most important development that has an extremely wide range of applications from TV remote controland cordless phones to cellular phones and satellite-based TV systems. It changedpeople's life style in every aspect. Especially during the last decade, the mobile radiocommunications industry has grown by an exponentially increasing rate, fueled bythe digital and $\mathrm{RF}$ (radio frequency) circuits design, fabrication and integration techniques and more computing power in chips. This trend will continue with an evengreater pace in the near future.

One natural question is: howcan we put high-rate data streams over radio links to satisfy our needs? New wirelessbroadband access techniques are anticipated to answer this question. For example,the coming $3 \mathrm{G}$ (third generation) cellular technology can provide us with up to $2 \mathrm{Mbps}$ (bits per second) data service. But that still does not meet the data rate required bymultimedia media communications like HDTV (high-definition television) and videoconference. Recently MIMO-OFDM systems have gained considerable attentions fromthe leading industry companies and the active academic community.A collection of problems including channel measurements and modeling, channel estimation, synchronization, IQ (in phase-quadrature)imbalance and PAPR (peak-to-average power ratio) have been widely studied by researchers.

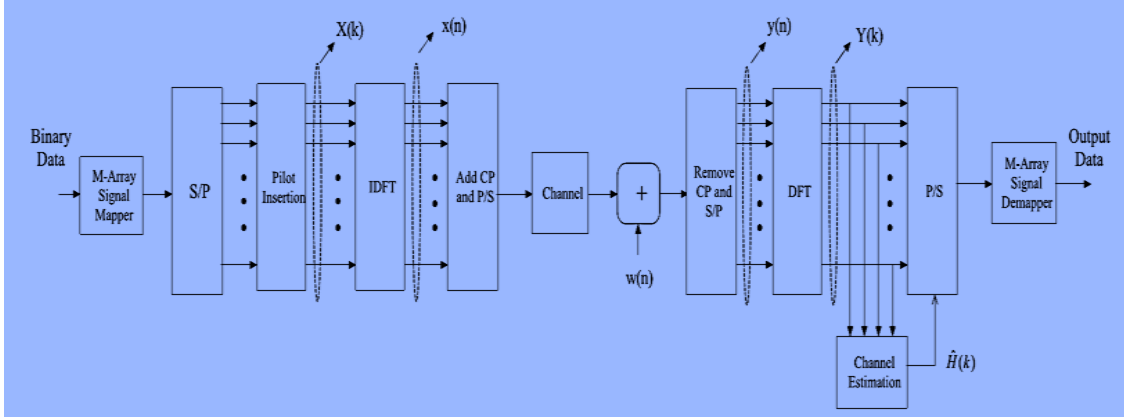

Figure 1.0: OFDM System Block Diagram 


\section{System Model}

The OFDM technology is widely used in two types of working environments, i.e., a wired environment and a wireless environment. When used to transmit signalsthrough wires like twisted wire pairs and coaxial cables, it is usually called as DMT (digital multi-tone). For instance, DMT is the core technology for all the xDSL (digital subscriber lines) systems which provide high-speed data service via existingtelephone networks. However, in a wireless environment such as radio broadcastingsystem and WLAN (wireless local area network), it is referred to as OFDM. Since weaim at performance enhancement for wireless communication systems, we use the termOFDM throughout this thesis. Furthermore, we only use the term MIMO-OFDMwhile explicitly addressing the OFDM systems combined with multiple antennas atboth ends of a wireless link. The history of OFDM can all the way date back to the mid 1960s, when Chang [2] published a paper on the synthesis of bandlimited orthogonal signals for multichanneldata transmission. He presented a new principle of transmitting signals simultaneously over a bandlimited channel without the ICI and the ISI. Right after Chang'spublication of his paper, Saltzburg [3] demonstrated the performance of the efficientparallel

With OFDM systems getting more popular applications, the requirements for abetter performance is becoming higher. Hence more research efforts are poured intothe investigation of OFDM systems. Pulse shaping $[7,8]$, at an interference pointview, is beneficial for OFDM systems since the spectrum of an OFDM signal can be shaped to be more well-localized in frequency; Synchronization $[9,10,11]$ in timedomain and in frequency domain renders OFDM systems robust against timing errors, phase noise, sampling frequency errors and carrier frequency offsets; For coherentdetection, channel estimation [46, 49, 48] provides accurate channel state informationto enhance performance of OFDM systems; Various effective techniques are exploitedto reduce the relatively high PAPR $[12,13]$ such as clipping and peak windowing. The principle of OFDM is to divide a single highdata-rate stream into a number oflower rate streams that are transmitted simultaneously over some narrower subchannels. Hence it is not only a modulation (frequency modulation) technique, but alsoa multiplexing (frequency-division multiplexing) technique. Before we mathematically describe the transmitter-channelreceiver structure of OFDM systems, a coupleof graphical intuitions will make it much easier to understand how OFDM works.OFDM starts with the $1 \mathrm{O}^{\prime}$, i.e., orthogonal. That orthogonality differs OFDM fromconventional FDM (frequency-division multiplexing) and is the source where all theadvantages of OFDM come from. The differencebetween OFDM and conventionalFDM is illustrated in Figure 1.1.
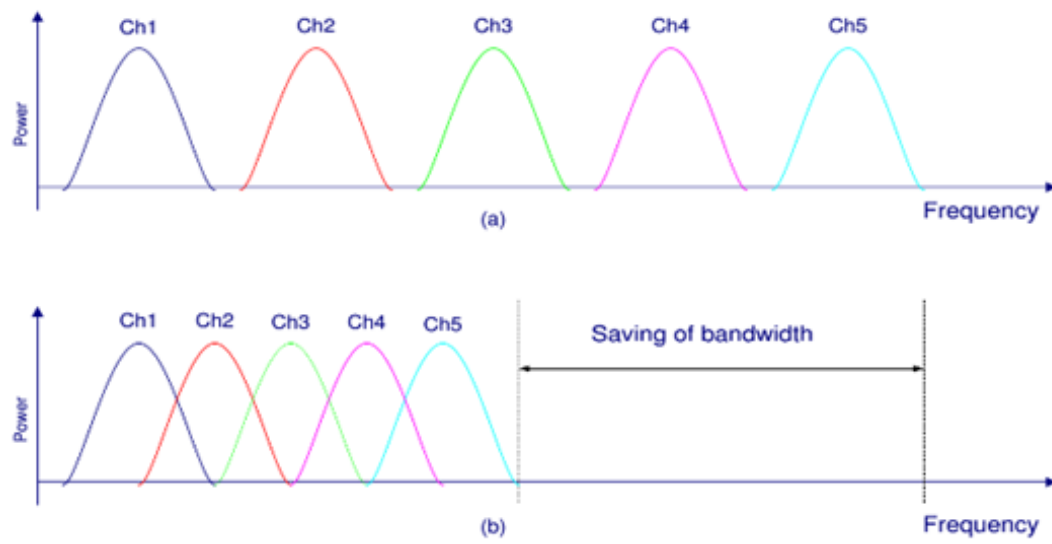

Figure 1.1: Comparison between conventional FDM and OFDM

\section{Motivation}

The sparse common support (SCS) model [6,2] is a reasonable assumption for many real-world channels. Sparsity is often observed inmultipathenvironments, whereeachindividualpathgivesrisetoan impulse in the channel response function [1,7]. The common support assumption is relevant when the distances between sensors are much smallerthandistancetraveledbytheelectromagnetic(orsound)wave in a time related to the inverse signal bandwidth (see [2] for a more detailed justification). In this case, certain frequency subbands of the channel response functions are well approximated by the SCS model, even though the full channel response functions might not agree with this assumption. First, the proposed scheme can achievesuper-resolution estimates of arbitrary path delays, which ismore suitable for wireless channels in practice. Second, dueto the small scale of the transmit and receive antenna arrayscompared to the long signal transmission distance in typicalMIMO antenna geometry, channel impulse responses (CIRs)of different transmit-receive antenna pairs share common pathdelays [5], which can be translated as a common sparse patternof CIRs due to the spatial correlation of MIMO channels.Meanwhile, such common sparse pattern is nearly unchangedalong several adjacent OFDM symbols due to the temporal correlation of wireless channels [6], [7]. Compared withprevious 
work which just simply extends the sparse channelestimation scheme in single antenna systems to that in MIMOby exploiting the spatial correlation of MIMO channels [5] or only considers the temporal correlation for single antennasystems [6], [7], the proposed scheme exploits both spatialand temporal correlations to improve the channel estimationaccuracy. Third, we reduce the pilot overhead by using thefinite rate of innovation (FRI) theory [8], which can recover theanalog sparse signal with very low sampling rate, as a result,the average pilot overhead per antenna only depends on thechannel sparsity level instead of the channel length.

\section{Mimo-OFDM Channel Estimation}

With the ever increasing number of wireless subscribers and their seemingly lgreedy"demands for high-data-rate services, radio spectrum becomes an extremely rare andinvaluable resource for all the countries in the world. Efficient use of radio spectrumrequires that modulated carriers be placed as close as possible without causing anyICI and be capable of carrying as many bits as possible. Optimally, the bandwidth ofeach carrier would be adjacent to its neighbors, so there would be no wasted bands.In practice, a guard band must be placed between neighboring carriers to providea guard space where a shaping filter can attenuate a neighboring carrier's signal.These guard bands are waste of spectrum. In order to transmit high-rate data, shortsymbol periods must be used. The symbol period Tsymis the inverse of the basebanddata rate $R(R=1=T s y m)$, so as $R$ increases, Tsymmust decrease. In a multipathenvironment, however, a shorter symbol period leads to an increased degree of ISI, and thus performance loss. OFDM addresses both of the two problems with itsunique modulation and multiplexing technique. OFDM divides the high-rate streaminto parallel lower rate data and hence prolongs the symbol duration, thus helpingto eliminate ISI. It also allows the bandwidth of subcarriers to overlap without ICIas long as the modulated carriers are orthogonal. OFDM therefore is considered asa good candidate modulation technique for broadband access in a very dispersiveenvironments [42, 43]. However, relying solely on OFDM technology to improve the spectral efficiencygives us only a partial solution. At the end of 1990s, seminal work by Foshini andGans [21] and, independently, by Teltar [22] showed that there is another alternativeto accomplish high-data-rate over wireless channels: the use of multiple antennasat the both ends of the wireless link, often referred to as MA (multiple antenna) orMIMO in the literature $[21,22,17,16,25,26]$. The MIMO technique does not requireany bandwidth expansions or any extra transmission power. Therefore, it provides apromising means to increase the spectral efficiency of a system. In his paper aboutthe capacity of multi-antenna Gaussian channels [22], Telatar showed that given awireless system employing NtTX (transmit) antennas and $\mathrm{Nr} \mathrm{RX}$ (receive) antennas, the maximum data rate at which error-free transmission over a fading channelis theoretically possible is proportional to the minimum of $\mathrm{Ntand} \mathrm{Nr}$ (provided thatthe $\mathrm{NtNr}$ transmission paths between the TX and RX antennas are statistically independent). Hence huge throughput gains may be achieved by adopting NtXNrMIMO systems compared to conventional $1 £ 1$ systems that use single antenna atboth ends of the link with the same requirement of power and bandwidth. Withmultiple antennas, a new domainnamely, the spatial domain is explored, as opposedto the existing systems in which the time and frequency domain are utilized.

\section{Signal Model}

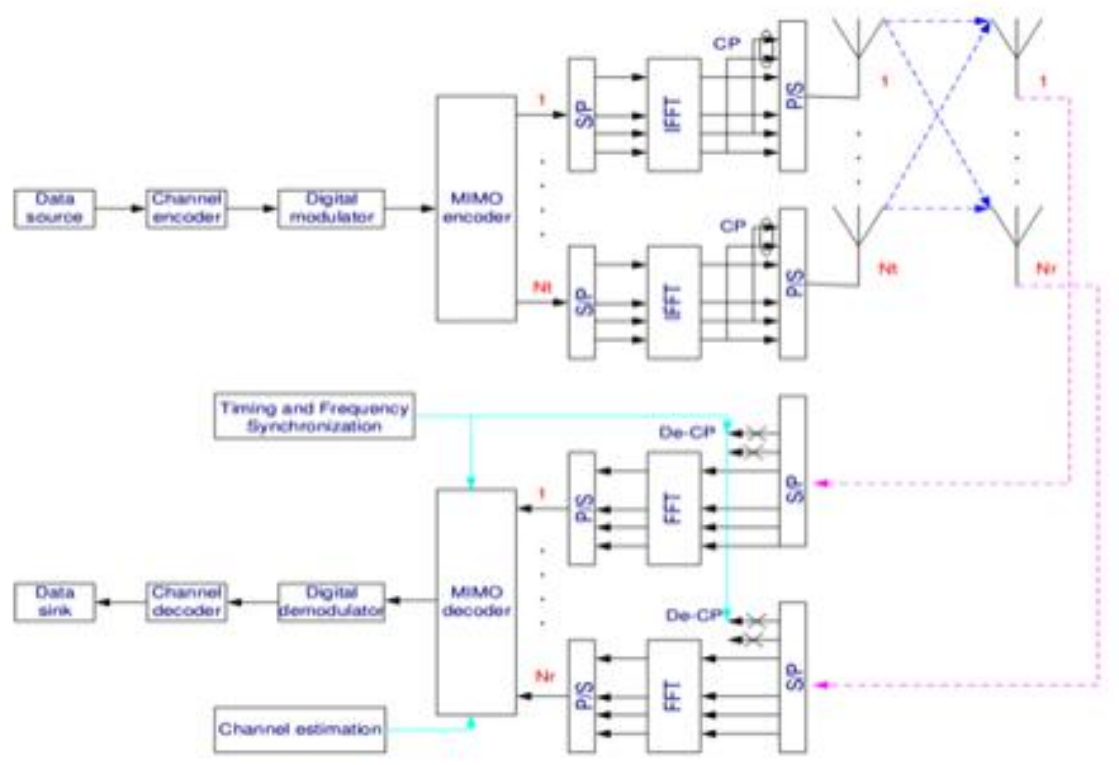

Figure 2.0: Ntx Nr MIMO-OFDM System model 


\section{Pilot-tone Design}

In order to have a simple and efficient LS algorithm for channel estimation, we have to design the square matrix $\mathrm{S}(\mathrm{P})(\mathrm{m})$ deliberately. In this section, the design will be illustrated by a theorem and an example.

The preamble design discussed in [50] adopted Tarokh's approach [18] to spacetime blockcode construction. It could be related to orthogonal design to whichour pilot-tone design also has a connection. In each of the firstNttraining blocksin a frame, a group of at least L pilot-tones are equally-placed and all the othertones are set to zeros. LS channel estimation can then be obtained based on theknown pilot-tones. The channel is assumed to be unchanged for the rest of the wholeframe. In a mobile environment, however, we cannot guarantee that the channel stateinformation estimated at the m-th block still holds true at the $(\mathrm{m}+\mathrm{Nt})$-th block.

Hence the preamble design in [50] is not suitable to be applied to the fast time-varyingchannels. In addition to this common disadvantage, the training sequences designedin [48] have to satisfy a condition called local orthogonality. It requires that, for theNtdifferent training sequences with length $\mathrm{N}$, they are orthogonal over the minimumset of elements for any starting position. The pilot design proposed in this paper aimsto remove the disadvantage and the constraint mentioned above. It actually has itsroots to Table I in [16], but it is notimplemented in space and time domain. On thecontrary, it is accomplished in space and frequency domain. We explicitly connectpilot-tone design with space-frequency coding so that we have more insights on itsdesign. Denote EPas the fixed total power for all the pilot-tones at each transmitantenna. Then the power allocated on each pilot-tone isEPNtLsince pilot-tones areall equalspaced and equalpowered. In some systems, the power of those pilot-tonescould be larger than the power of data symbols for a better estimation of the wirelesschannel. We assume in our work that the pilot-tones and other data are all equallynormalized such that the average power for all different mappings is the same. Ourpilot-tone design is illustrated in the following theorem.

Theorem 2.1 Let $S_{\text {diag }, j}^{p i}(m)=\alpha_{p i, j} I_{L N_{r}}|\alpha p i, j|=\sqrt{\frac{E_{P}}{N_{t} L}}, i, j=1,2, \ldots, N_{t}$, then

$\frac{1}{\sqrt{E_{P}}} S^{p}(m)$ is a unitary matrix if

$S_{S F C}^{(P)}(m)=\sqrt{\frac{L}{E_{P}}}\left[\begin{array}{l}S_{\text {diag }, 1}^{(p i)}(m) \ldots S_{\text {diag }, N_{t}}^{p i}(m) \\ \cdot \\ S_{\text {diag }, 1}^{(p i)}(m) \ldots S_{\text {diag }, N_{t}}^{p i}(m)\end{array}\right]$

is a unitary matrix

\section{Results}

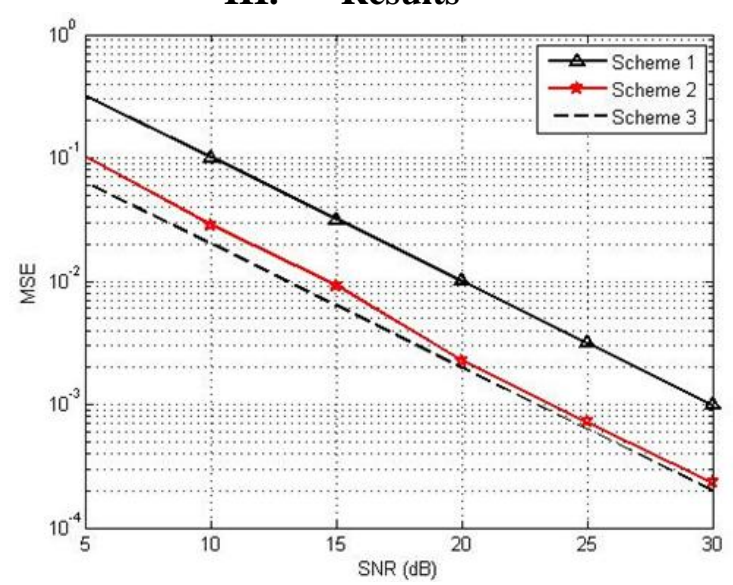

Figure 2.1 MSE vs SNR 


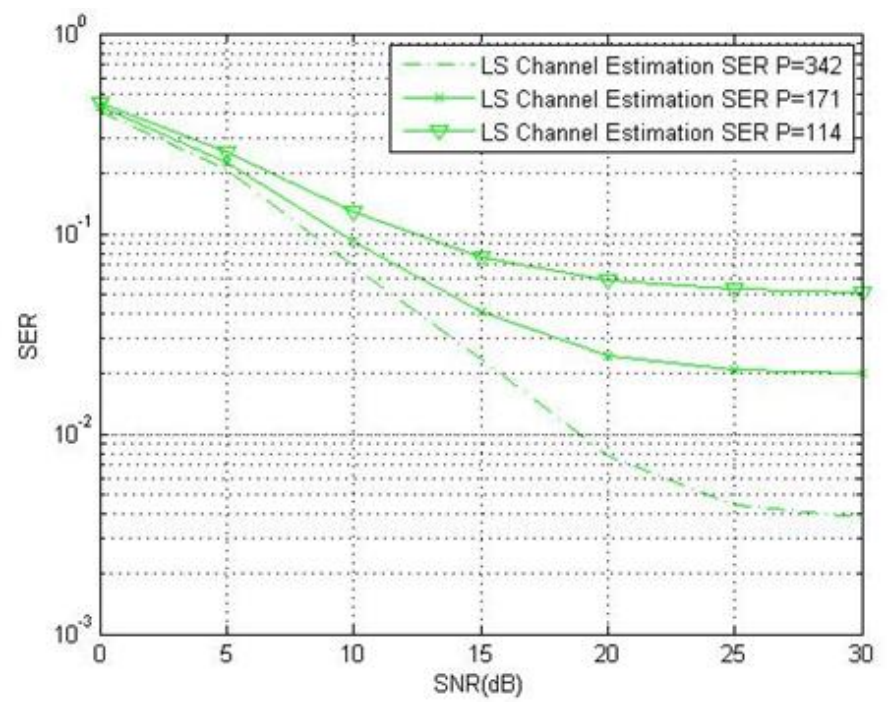

Figure 2.2 LS Estimation

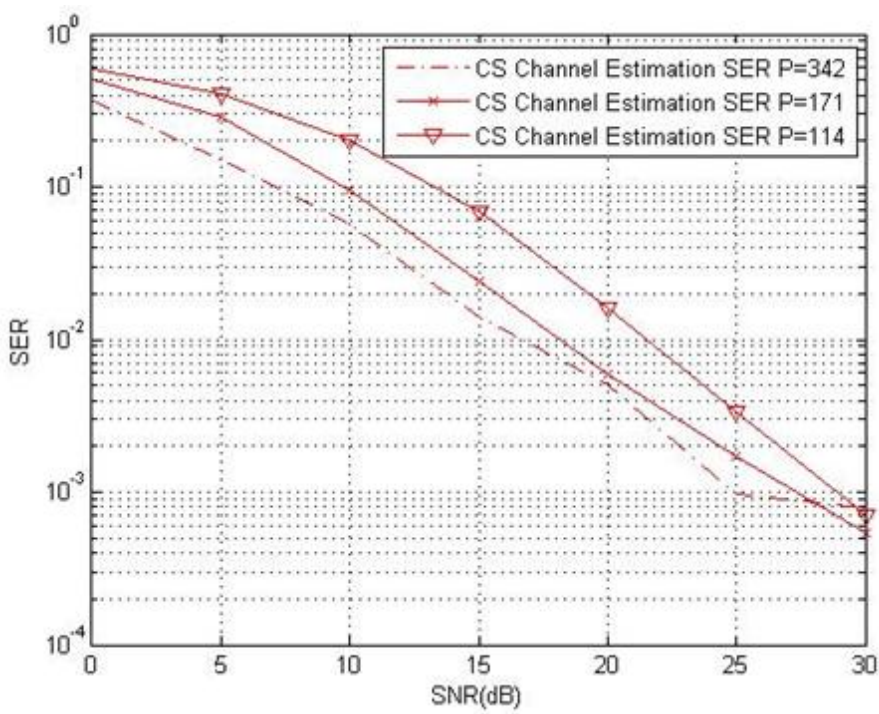

Figure 2.3 CS Estimation

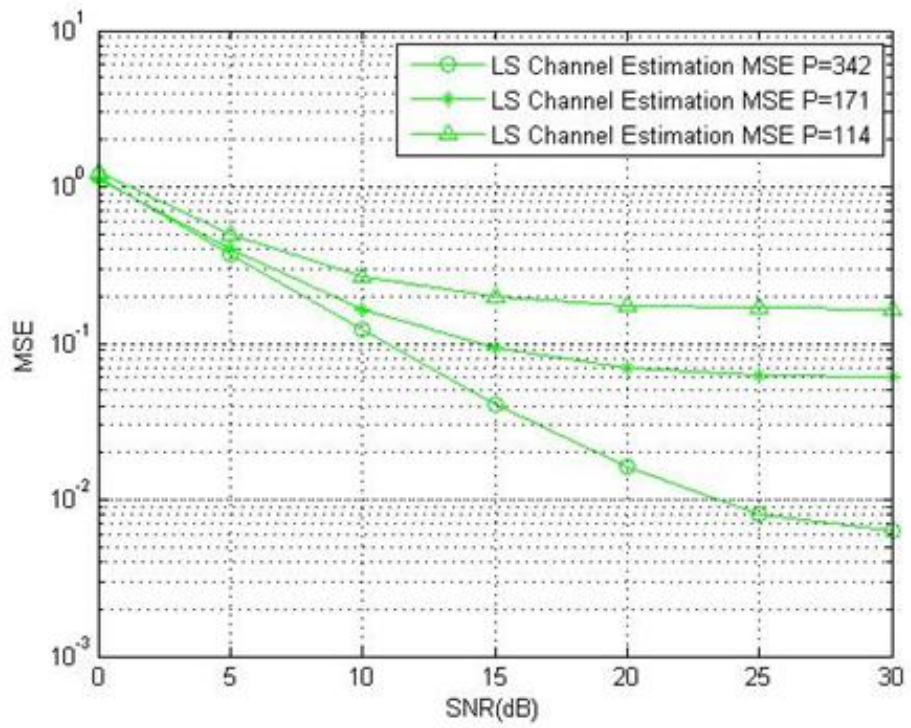

Figure 2.4 CS Estimation 


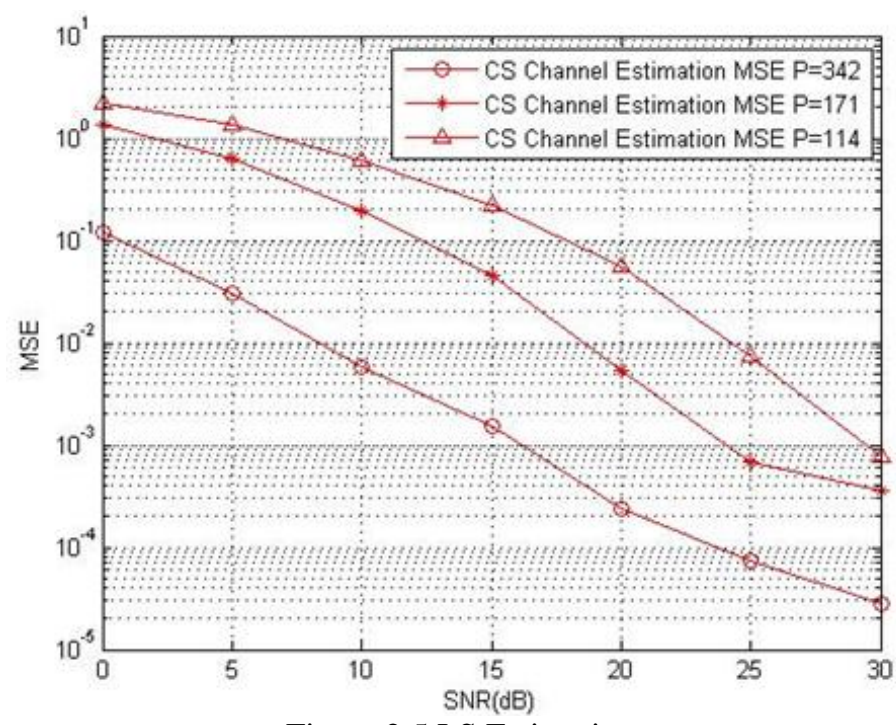

Figure 2.5 LS Estimation

\section{Conclusion}

This paper, in the first part, addresses the problem of channel estimation ofMIMO-OFDM systems. It starts from the matrix representation of the signal modelof MIMO-OFDM systems, which clearly describes the relation of signals in frequencydomain and time domain and expressing operations like adding $\mathrm{CP}$ and removingCP as matrix product. From the resulting MIMO-OFDM signal model, a pilot tonebased channel estimation is proposed to estimate the fast time-varying and frequencyselective fading channel via the leastsquares method. The least-squares is selectedfor the purpose of low complexity, though some other methods such as MMSE andML may produce better estimation performance. To further reduce the computational complexity, the pilot tone matrix is designed as a unitary matrix to save thecomputation of the matrix inversion in the standard LS solution. The pilot tonematrix is designed in a simple way that Ntdisjoint pilot tone sets are placed at oneOFDM block on each transmit antenna. Each pilot tone set has L pilot tones whichare equallyspaced and equally-powered. By choosing the pilot tones based on our de-. sign, those pilot tones comprise a unitary matrix. For a simple $2 £ 2$ case, Alamouti'sorthogonal structure is exploited. And the design can be readily extended to a con $^{-}$gurable MIMO-OFDM system with any number of transmit and receive antennas. Fora ${ }^{-}$xed power of pilot tones, our design can be proved to be also optimal in the senseof achieving the minimum MSE of channel estimation. Compared with some relativepilot tone designs in the literature, our channel estimation method di®ers in its abilityto estimate fast time-varying wireless channel since pilot tones are inserted into eachOFDM block, and in its explicit relation with space-frequency code design which canbene $e^{-}$the channel estimation in return. Seeking for a robust channel estimator withlower complexity for MIMO-OFDM systems, we are looking at the following aspectsin the future.

\section{References}

[1]. Z. G. Karabulut and A. Yongacoglu, "Sparse channel estimation using orthogonal matching pursuit algorithm," 2004 IEEE 60th Vehicular Technology Conference, vol. 60(6), pp. 3880-3884, 2004.

[2]. J. A. Tropp and A. C. Gilbert, "Signal recovery from random measurements via orthogonal matching pursuit," IEEE Transaction on Information Theory, vol. 53(12), pp. 4655-4666, 2007.

[3]. U. W. Bajwa, J. Haupt, G. Raz, and R. Nowak, "Compressed channel sensing," To appear in Proc. 42ndAnnu. Conf. Information Sciences and Systems (CISS'08), Mar. 19-21, 2008.

[4]. D. L. Donoho, M. Elad, and V. N. Temlyakov, "Stable recovery of sparse overcomplete representations in the presence of noise," IEEE Transaction on Information Theory, vol. 52(1), pp. 6-18, Jan. 2006.

[5]. E. J. Candès, "The restricted isometry property and its implications for compressed sensing," CompteRendus de l'Academie des Sciences, Paris, vol. Serie I, 346, pp. 589-592, 2008.

[6]. S. Kunis and H. Rauhut, "Random sampling of sparse trigonometric polynomials II - orthogonal matching pursuit versus basis pursuit," Found. Comput. Math., vol. 8, pp. 737-763, Dec. 2008.

[7]. E. Candès and T. Tao, "TheDantzig selector: Statistical estimation when p is much larger than n," Annals of Statistics, vol. 35, pp. 2392-2404, 2006.

[8]. N. H. Nguyen and T. D. Tran, "The stability of regularized orthogonal matching pursuit algorithm," http://www.dsp.ece.rice.edu/cs/Stability_of_ROMP.pdf. 